Article type : Editorial

\title{
Advice Regarding Alcohol Use by Individuals With Nonalcoholic Fatty Liver Disease: Primum non nocere
}

\author{
Winston Dunn ${ }^{1}$ and Naga Chalasani ${ }^{2}$ \\ ${ }^{1}$ The University of Kansas Medical Center, Kansas City, KS \\ ${ }^{2}$ Indiana University School of Medicine, Indianapolis, IN
}

Abbreviations: ADH, alcohol dehydrogenase; AUD, alcohol use disorder; NAFLD, nonalcoholic fatty liver disease.

Conflicts of Interest: Nothing to report

Funding: Supported in part by 1 K23 DK109294 to W.D.

\author{
Corresponding Author \\ Naga Chalasani, M.D. \\ Indiana University School of Medicine \\ 702 Rotary Circle, Suite 225 \\ Indianapolis, IN 46202 \\ Fax +1-317-278 1949 \\ E-mail: nchalasa@iu.edu
}

Individuals with nonalcoholic fatty liver disease (NAFLD) often ask their health care providers about routinely consuming non-heavy amounts of alcohol for pleasure and potential health benefits. According to the American Association for the Study of Liver Diseases (AASLD) Practice Guidance published in 2018, there are insufficient data to make a recommendation with regard to nonheavy alcohol consumption by individuals with NAFLD. ${ }^{(1)}$ Although several cross-sectional studies 
have suggested that light alcohol consumption (on average, $<1$ drink per day) may have a beneficial effect on the presence and severity of NAFLD, a large meta-regression analysis suggested that lower body mass index among light and moderate drinkers is a potential confounder and thus casts doubt on the rigor of those studies. ${ }^{(2)}$ The AASLD Practice Guidance also pointed out that there have been no longitudinal studies examining the impact of non-heavy alcohol consumption on the natural history of liver disease and its extrahepatic harms and benefits in individuals with established NAFLD. Similarly, in a critical review, Ajmera and colleagues concluded that a strong recommendation favoring the benefits of moderate alcohol use in NAFLD cannot be made due to significant methodological limitations of published studies (e.g., incomplete adjustment for confounders). ${ }^{(3)}$

In this issue of HEPATOLOGY, ${ }^{(4)}$ Chang and colleagues report the results of their study, which examined the relationship between non-heavy alcohol consumption and the risk of progression from low risk to intermediate and high risk for advanced fibrosis categories based on noninvasive fibrosis indices (NAFLD fibrosis score [NFS] FIB-4 and AST-to-platelet ratio index [APRI]) in 57,254 Korean men and women with NAFLD.$^{(3)}$ After adjusting for potential confounders, compared to nondrinkers, light and moderate drinkers were significantly more likely to transition from low risk to intermediate or high risk for advanced fibrosis categories. For example, compared to nondrinkers, moderate drinkers at low risk for advanced fibrosis at baseline were $40 \%$ more likely to transition into moderate to high risk for advanced fibrosis category over a median follow-up of 8.3 years. Based on these results, the authors concluded that non-heavy alcohol consumption was significantly and independently associated with worsening of noninvasive markers of fibrosis, indicating that even moderate alcohol consumption might be harmful in individuals with NAFLD. A major limitation of this study is that the validity of serial FIB-4 and NFS measurements for characterizing the natural history of fibrosis in NAFLD is largely unknown. This observation is corroborated by another interesting paper that reported the results of a Mendelian randomization study with rs1229984 G>A variant in the alcohol dehydrogenase $1 \mathrm{~B}(A D H 1 B)$ gene as a proxy for long-term alcohol exposure (individuals with ancestral $\mathrm{G}$ allele are likely to drink more, and those with rare A allele are likely to drink less). ${ }^{(5)}$ In this study, individuals with NAFLD and $A D H 1 B G / G$ who drank only $7.5 \mathrm{~g}$ of alcohol on average per day had significantly higher steatosis, necroinflammation, and NAFLD activity score compared to individuals with NAFLD and $A D H 1 B A / G$ or $A / A$ who drank much less alcohol, suggesting that even light alcohol consumption can be associated with more severe liver histology in NAFLD. In a paired liver biopsy study from the NASH Clinical Research Network, compared to 117 nondrinkers, 168 individuals with modest alcohol use ( $\leq 2$ drinks per day) were less likely to experience spontaneous improvement in liver histology during a mean follow-up of 47 months between biopsies. ${ }^{(6)}$ Although many studies have shown that the prevalence of NAFLD is 
lower with moderate alcohol consumption compared to no alcohol consumption in the general population, these studies are not directly applicable to patients who have existing NAFLD. ${ }^{(3)}$

Even though there is heightened publicity around the potential benefits of light to moderate alcohol drinking on cardiovascular and diabetes risks, the epidemiological evidence linking light drinking to increased risk of certain types of solid tumors unfortunately evades any serious scrutiny. In a recent meta-analysis consisting of 222 publications and 92,000 light drinkers and 60,000 nondrinkers with cancers, ${ }^{(7)}$ light drinking was associated with an increased risk for oropharyngeal cancer (RR [relative risk], 1.17; 95\% CI: 1.06-1.29), esophageal squamous cell cancer (RR, 1:30; 95\% CI: 1.09-1.56), and female breast cancer (RR, 1.05; 95\% CI: 1.02-1.08) but not liver (RR, 1.03; 95\% CI: 0.90-1.17) or colorectal cancer (RR, 0.99; 95\% CI: 0.96-1.04). In fact, the European Code Against Cancer, 4th edition, recommends that "If you drink alcohol of any type, limit your intake. Not drinking alcohol is better for cancer prevention." ${ }^{(8)}$ We believe this recommendation is readily applicable to our patients with NAFLD who are at increased risk for cancers because of obesity and diabetes, two commonly recognized risk factors for cancers.

Recent trends of alcohol consumption in the United States are quite troubling and yet are not receiving sufficient attention. The results from the National Epidemiologic Survey on Alcohol and Related Conditions showed that the frequency of high-risk drinking and Diagnostic and Statistic Manual of Mental Disorders, 4th Edition (DSM-IV) alcohol use disorder (AUD) has increased by $29.9 \%$ and 49.4\%, respectively, between 20012002 and 20122013. ${ }^{(9)}$ While virtually all age, geographic, and socioeconomic groups were affected by these increases, striking increases were noted in women who exhibited a 58\% increase in high-risk drinking and $84 \%$ increase in AUD during that 10-year time frame. According to the National Institute on Alcohol Abuse and Alcoholism Surveillance Report No. 105, age-specific death rates from alcohol-related cirrhosis rose dramatically between 2009 and 2013 for the first time since 1973. ${ }^{(10)}$ Although the age-adjusted death rate from alcohol-related cirrhosis between 2000 and 2013 has increased by $19.1 \%$ for white men and 50\% for white women, it has intriguingly decreased for both black men and women by approximately $32 \%$. Tapper and Parikh ${ }^{(11)}$ recently reported that mortality due to alcoholic cirrhosis has been increasing in the United States since 2009, with people aged 25 to 34 years exhibiting the greatest increase (average annual increase of $10.5 \%$ between 2009 and 2016). These sobering trends demand us to be far more attentive to the harmful effects of alcohol consumption.

This article is protected by copyright. All rights reserved. 
Unless newer data showing a clearly favorable benefit-to-risk ratio of non-heavy drinking in individuals with NAFLD become available, the observations by Chang et al. ${ }^{(4)}$ and Ajmera et al. ${ }^{(6)}$ should compel us to advise our patients with NAFLD against regular consumption of alcohol, even in non-heavy amounts, because it is our duty to first do no harm.

\section{References}

1) Chalasani N, Younossi Z, Lavine JE, Charlton M, Cusi K, Rinella M et al. The diagnosis and management of nonalcoholic fatty liver disease. Practice guidance by the American Association for the Study of Liver Diseases, American College of Gastroenterology, and American Gastroenterology Association. HePATOLOGY 2018;68:361-371.

2) Sookoian S, Castano GO, Pirola CJ. Modest alcohol consumption decreases the risk of nonalcoholic fatty liver disease: A meta-analysis of 43,175 individuals. Gut 2014;63:530-532.

3) Ajmera VH, Terrault NA, Harrison SA. Is moderate alcohol use in nonalcoholic fatty liver disease good or bad? A critical review. HEPATOLOGY 2017;65:2090-2099.

4) Chang Y, Cho YK, Kim Y, Sung E, Ahn J, Jung HS, et al. Non-heavy drinking and worsening of non-invasive fibrosis markers in nonalcoholic fatty liver disease: A cohort study. HEPATOLOGY 2018 July 17. [Epub ahead of print]

5) Sookoian S, Flichman D, Castano GO, Pirola CJ. Mendelian randomisation suggests no beneficial effect of moderate alcohol consumption on the severity of nonalcoholic fatty liver disease. Aliment Pharmacol Ther 2016;44:1224-1234.

6) Ajmera V, Belt P, Wilson LA, Gill RM, Loomba R, Kleiner DE, et al. Among patients with nonalcoholic fatty liver disease, modest alcohol use is associated with less improvement in histologic steatosis and steatohepatitis. Clin Gastroenterol Hepatol 2018 March 14. [Epub ahead of print]

7) Bagnardi V, Rota M, Botteri E, Tramacere I, Islami F, Fediriko V, et al. Light alcohol drinking and cancer: A meta-analysis. Ann Oncol 2013;24:301-308.

8) Scocciianti C, Cecchini M, Anderson AS, Berino F, Boutron-Ruault MC, Espina C, et al. European code against cancer 4th edition: alcohol drinking and cancer. Cancer Epidemiol 2015;395:S67-S74.

9) Grant BF, Chou SP, Saha TD, Pickering RP, Kerridge BT, Ruan WJ, et al. Prevalence of 12Month Alcohol Use, High-Risk Drinking, and DSM-IV Alcohol Use Disorder in the United States, 
2001-2002 to 2012-2013: Results From the National Epidemiologic Survey on Alcohol and Related Conditions. JAMA Psychiatry 2017;74:911-923.

10) Yoon YH, Chen CM. Liver cirrhosis mortality in the United States: national, state, and regional trends, 2000-2013. https://pubs.niaaa.nih.gov/publications/surveillance105/Cirr13.pdf. Accessed August 11, 2018.

11) Tapper EB, Parikh ND. Mortality due to cirrhosis and liver cancer in the United States, 19992016: observational study. BMJ 2018;362:k2817. doi:10.1136/bmj.k2817.

This article is protected by copyright. All rights reserved. 